\title{
Understanding the Loop Quantum Cosmology and the Concept of Time
}

\author{
Bhuvaneshwari Kashi ${ }^{a, *}$ \\ ${ }^{a}$ CVR College of Engineering, \\ Ibrahimpatnam, Telangana, India \\ E-mail: bhuvana9kashi@gmail.com
}

\begin{abstract}
Time is an enigmatic property of the universe which confounded physicists for ages. This property is increasingly dark and cryptic when we deal with metric spaces of the microscopic scales in the universe. There are remarkable theories, in particular, the loop quantum gravity (LQG) which helps us in understanding the cosmology of these microscopic scales. However, the theory possesses considerable complications in explaining the concept of time. We redress the notions of quantum cosmology and the loop quantum gravity. The elegance of the theory in describing the microscopic scales is discussed. We try to emphasise the concept of the time interpreted as far as the notion of quantum gravity is concerned. We aim to review and re-analyse the loop gravity, the perception of the dynamic time and the timeless universe.
\end{abstract}

\footnotetext{
*** The European Physical Society Conference on High Energy Physics (EPS-HEP2021), *** *** 26-30 July $2021 * * *$

*** Online conference, jointly organized by Universität Hamburg and the research center DESY ***
}

\footnotetext{
${ }^{*}$ Speaker
} 


\section{Loop Quantum Gravity - a concise note}

The black hole's gravitational field's quantum effects lead it to radiate energy as if it were hot, revealing a close relationship between quantum theory, gravity, and thermodynamics. Theorists with this clue have been searching for something more fundamental than space and time for centuries. Loop quantum gravity is one of the possible solutions (LQG). This approach was proposed at a conference in India in the year 1987. It is a quantum gravity candidate which currently competes with string theory. LQG is a quantum gravity theory in which the structure of space is made up of finite loops woven together in a network. In [3]LQG the space and time are considered as quantized quantities. It is one of the best way to describe quantum space time from a non-pertubative and background independent perspective. It is the Hilbert space containing the transition amplitudes. Loop variables (which are essentially related to Yang Mill's variables) are included in the loop quantum gravity, which tend to grow out of Ashtekar's new variables. They are $: E_{i}^{a}(x):=\sqrt{h}(x) e_{i}^{a}(x)$ where the configuration variable is the connection $G A_{a}^{i}(x)=\Gamma_{i}^{a}(x)+_{a}^{i}(x)$. There are the canonical approach and the covariant approach i.e, the spin foam approach of LQG. We use spin foam formalism [1] of LQG to understand quantum gravity.

\section{Quantum Cosmology and LQG}

Classical cosmology is based on Albert Einstein's general theory of relativity, which he proposed in 1915. Here the universe is considered to be a space-time fabric. It proposes that the space time is physical and dynamic. The spacial homogenity and the isotropy implies that the metric has FLRW form.

$$
d s^{2}=-d t^{2}+a^{2}(t) d x^{-2}
$$

The theory of general theory of relativity becomes unreliable when the energy density and curvature approaches the Planck scale. GR is not perturbatively renormalisable. [5] Thus the quantum aspects of the gravitational degrees of freedom are expected to govern that regime. The general theory of relativity is insufficient to address the quantum nature of the space and time. Quantum cosmology is today a theoretical effort to understand the quantum nature of the universe. [4] refers that quantum cosmology is manifestation of quantum descriptions of all kinds of matter fields and their interactions. This involves quantizing chunks of amorphous and homogeneous space as a first approximation of "space-time atom". In 1969 Charles Misner introduced the concept of quantum cosmology by truncating the Wheeler-De-Wit equation(WDW) to finite number of degrees of freedom. As there are only finite number of degrees of freedom a(t), $\phi(t)$, field theoretical difficulties are by passed and the analysis is reduced to standard quantum mechanics.

$$
\begin{gathered}
{\left[-G_{i j k l} \frac{\delta^{2}}{\delta_{\gamma_{i j}} \delta_{\gamma_{k l}}}-{ }^{3} R(\gamma) \gamma^{\frac{1}{2}}+2 \Lambda \gamma^{\frac{1}{2}}\right] \psi\left[\gamma_{i j}\right]=0} \\
l_{P 1}^{4} \frac{\partial^{2}}{\partial a^{2}}(f(a) \psi(a, \phi))=\mathrm{const} G \quad \hat{H}_{\phi} p \operatorname{si}(a, \phi)
\end{gathered}
$$

The (2) explains the quantum evolution governed by WDW theory with the quantum states $\psi(a, \phi) ; \hat{a} \psi(a, \phi)=a \psi(a, \phi)$. 
Unlike the classical cosmology, in quantum cosmology, here we consider the universe as a wave function. This theory is based on the theory of quantum gravity. There are a number of theories that aim to explain quantum gravity and cosmology, the most well-known of which are string theory and LQG. When LQG is used to quantum cosmology, it is referred to as Loop Quantum Cosmology with certain bounds.

\subsection{Loop Quantum Cosmology}

In WDW equation we donot have well structured kinematics. The LQG when applied provides a background independent frame work i.e, the differential invariance prefers where the kinematical framework. We know that Hilbert space of LQC $H=L^{2}(\overline{\mathbb{R}} B o h r)$ is disimilar to the WDW theory. The WDW differential operator breaks down to ambiguity on it. Thus the WDW differential operator is replaced by a difference operator.

$$
C^{+}(v) \psi(v+4, \phi)+C(v) \psi(v, \phi)+C-(v) \psi(v-4, \phi)=\gamma l \quad{ }_{P}^{2} \hat{H}_{\phi} \psi(v, \phi)(*)
$$

where the step size in $\left(^{*}\right)$ is governed by the area gap of the LQG. This step size, the quantum gap which is ignored by the WDW theory is obtained in the LQC.

From [5] we are restating that when we applied LQG to the cosmological space time we obtain a resulting frame work i.e, the loop quantum cosmology (LQC). The problem of time and inner product; quantum probabilities; and other examples of how quantum gravity effects change classical dynamical equations have recently been used to better understand whether and how quantum gravitational effects avoid classical singularities; how a smooth continuum space-time emerges from the underlying quantum theory; and so on. According to the notion, the Big bounce occurs before the Big Bang.

\section{The notion of time}

In general when speak about time we refer to the occurrence of an event with respect to another. The notion of time in physics plays an important role. In many circumstances, time is assumed to move uniformly in a linear, forward-pointing path. We know that the Newtonian Space is considered as 3-dimensional Euclidean space and time as 1- dimensional. The Newtonian time suggests us that the time flows uniformly with no obligation of an event to occur. This becomes merely an approximation when the LQG is applied. Einstein's time is full of clocks and rods. In GR we have seen that the time is considered one of the aspects of the dynamical field, gravitational field.

$$
T=\int \sqrt{g_{\mu \nu} d x^{\mu} d x^{v}}
$$

In this scenario, LQG is similar to GR. The time is consider as many clock times measured by different clocks. Here we address an event A with respect to other event occurred i.e, event B. These events are thought to be temporarily adjacent. The spinfoams capture the network of temporal adjacency of elementary processes in the gravitational field. Interestingly there can occur quantum fluctuations and also there can be different super positions between different values of the same clock. From [2] The Hamilton-Jacobi formulation, which is a quantum counterpart of the classical GR formulation that does not use the time variable $t$ at all, explicitly determines the transition 
amplitudes between quantum states of the gravitational field. These can be used in conjunction with matter and clocks. The theory uses no preferred clock variable and all variables change at the same time. At the fundamental level of quantum gravity, it is frequently argued that "time does not exist" in this weak sense. The absence of a time variable in the fundamental equations is indicated by this phrase. This isn't to say that nature hasn't changed. This is expressed in terms of process probability amplitudes.

\section{Conclusion}

LQG is one of the best candidates of quantum gravity. LQG when meets quantum cosmology LQC evolves which describes the big bang as big bounce. Time is an enigmatic and cryptic property of the universe. We theorise that time is dynamic after looking at it through the lens of LQG. It's a web of interconnected quantum processes, each of which obeys probabilistic laws that the theory captures. The net of adjacent spacetime regions is linked with the net of quantum interactions between systems. Quantum gravity describes a universe that is neither flowing along a single time axis nor organised into a smooth Einsteinian geometry. It's a web of interconnected quantum processes, each of which obeys probabilistic laws that the theory captures. The net of adjacent spacetime regions is linked with the net of quantum interactions between systems.

\section{References}

[1] John C Baez 1998 Class. Quantum Grav. 15 1827, doi:10.1088/0264-9381/15/7/004

[2] Rovelli, C., "Space and Time in Loop Quantum Gravity", <i>arXiv e-prints</i>, 2018.

[3] Rovelli C. Loop Quantum Gravity. Living Rev Relativ. 1998;1(1):1. doi:10.12942/lrr-1998-1

[4] Martin Bojowald 2015 Rep. Prog. Phys. 78 023901, doi:10.1088/0034-4885/78/2/023901

[5] Agulló, Iván and Alejandro Corichi. "Loop Quantum Cosmology.” arXiv: General Relativity and Quantum Cosmology 140 (2013): 809-839. 